\title{
analyse critique des essais de cisaillement en mécanique des roches
}

\author{
par \\ Emanuele Fumagalli \\ Prof. Ing. Université de Bergame (Italie)
}

\section{Généralités sur la nature de la discontinuité}

II est reconnu que la stabilité des discontinuités est fondamentale pour la résistance et l'équilibre général d'un massif rocheux.

Ces discontinuités peuvent être de différente nature: failles tectoniques plus ou moins actives, ruptures, systèmes de diaclases, systèmes stratifiés de roches sédimentaires, cavernes, etc.

Lorsqu'elles résultent de la présence de diaclases ou de systèmes stratifiés sédimentaires, elles se présentent normalement sous forme de familles de discontinuités parallèles plus ou moins rapprochées.

Leur équilibre doit être examiné aussi bien sous le rapport des conditions de contact et de leur orientation, qu'en regard des systèmes de charges appliqués et des conditions de confinement (discontinuités affleurantes ou internes).

Notre analyse envisagera en particulier les conditions naturelles de contact.

On peut d'abord noter que les discontinuités ne constituent pas, en elles-mêmes, une cause de faiblesse à l'intérieur du massif rocheux où l'état du tenseur hydraulique ( $\left.{ }^{*}\right)$ est normalement de compression, et toujours d'une valeur considérable, au moins lorsque les deux conditions suivantes sont respectées :

1) l'angle de frottement entre les surfaces de discontinuité est du même ordre de grandeur que celui de la roche matrice, ce qui se produit d'ordinaire en cas de contact direct entre roches saines;

2) les surfaces de discontinuité sont en contact l'une de l'autre, autrement dit le système est relativement fermé et n'est troublé ni par d'importantes solutions de continuité, ni par des cavités naturelles ou artificielles.
Par contre, les discontinuités peuvent constituer un élément de faiblesse lorsqu'on est en présence d'intrusions (d'argile humide, par exemple) et que les surfaces de discontinuité affleurent : c'est le cas, par exemple, du contrefort rive gauche du modèle du barrage de Cà Selva (fig. 1). L'angle de frottement est alors considérablement réduit par rapport aux conditions de contact de la roche matrice.

Certaines conditions accidentelles d'instabilité en fonction principalement de l'orientation de la discontinuité et des systèmes de charges appliqués peuvent également se manifester au voisinage des surfaces libres où, justement, la valeur du tenseur hydraulique est pratiquement nulle. Cela peut se produire aussi bien sur les surfaces extérieures que sur les surfaces intérieures, en présence de cavités naturelles ou artificielles.

\section{Essais de cisaillement}

Plusieurs recherches ont été faites récemment pour étudier la résistance à la rupture par cisaillement le long des surfaces de discontinuité.

En examinant un diagramme de cisaillement $\tau-\Delta s$ pour des surfaces entre roches saines nous voyons que si l'on opère à $\sigma_{n}=$ constant, le diagramme croît jusqu'à atteindre une valeur $\tau_{\mathrm{p}}$ maximale ou de pic, puis décroît ensuite et se stabilise, avec une allure approximativement horizontale, pour une valeur $\tau_{\mathbf{r}}=$ constante appelée parfois résistance résiduelle.

(") On appelle ici «tenseur hydraulique » le tenseur sphérique d'intensité uniforme égale à la contrainte principale minimale. 


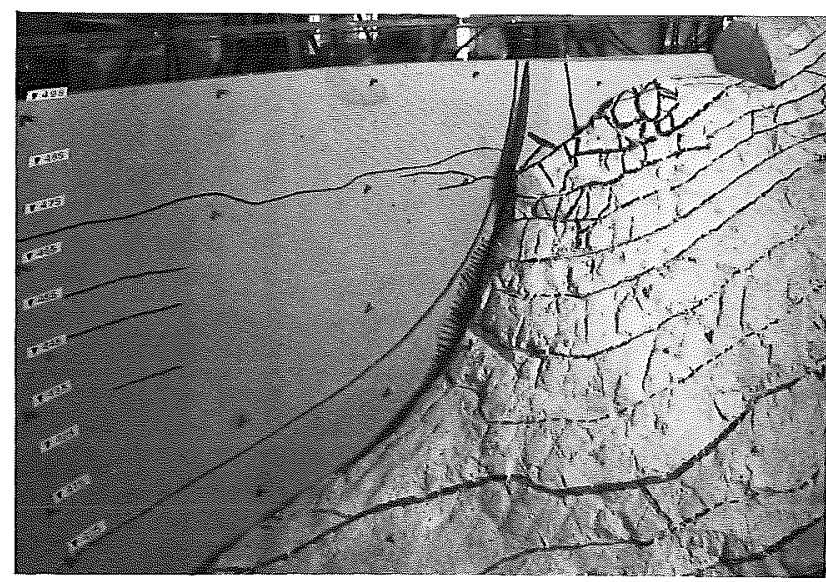

Fig. 1 Modèle du barrage de Cà Selva : glissements graduels des systèmes de couches affleurantes dans le massif rocheux d'appui en rive gauche
Les expérimentateurs ne sont pas entièrement d'accord sur les lois de variation de ces différentes grandeurs en présence de surfaces rugueuses. Mon expérience personnelle me fait considérer comme suffigamment appropriée aux problèmes pratiques l'interprétation des phénomènes selon les diagrammes de la figure $3 a$, où $K_{s}, \tau_{p}, \tau_{r}$ augmentent en fonction de $\sigma_{n}$, sans respecter une loi de proportionnalité.

Si les surfaces sont suffisamment lisses au début de l'essai (fig. 3b), la valeur de $\phi_{r}=\operatorname{arctg} \tau_{r} / \sigma_{n}$ est relativement constánte en fonction de $\sigma_{n}$; c'est justement vers cette valeur $\phi_{r}$ limite, que la courbe des $\phi_{r}$ correspondant aux surfaces rugueuses tend pour des $\sigma_{n}$ très élevées (fig. 4).

D'autre part, la différence $\tau_{\mathrm{p}}-\tau_{\mathrm{r}}$ tend d'autant plus à se réduire que les surfaces sont plus lisses; en d'autres termes, le pic s'aplatit au point de devenir difficile à identifier, alors que $K_{s}$ reste à peu près constant (fig. $3 b$ ).

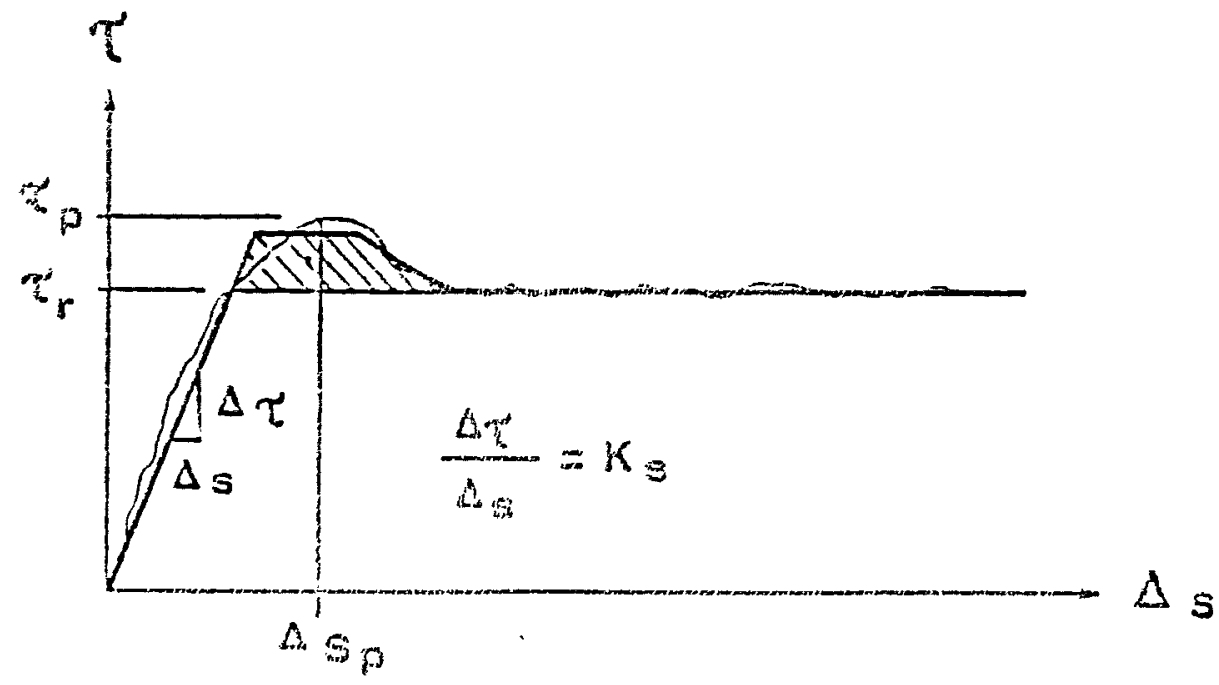

Fig. 2 Diagramme indicatif d'un essai de cisaillement $K_{s}=\Delta_{\tau} / \Delta_{s}$ (rigidité au cisaillement)

En schématisant la courbe (fig. 2) nous pouvons identifier un segment initial d'inclinaison $\Delta_{\tau} / \Delta_{s}=K_{s}$ représentant la rigidité au cisaillement, une valeur $\tau_{\mathrm{p}}$ maximale ou de pic, une zone de pic et, enfin, une valeur $\tau_{r}$, résistance limite ou de cisaillement résiduel $\left({ }^{*}\right)$.

(*) Les diagrammes représentés d'ordinaire par différents auteurs schématisent la courbe $\tau-\Delta_{\mathrm{s}}$ en identifiant un pic pointu. On préfère ici une schématisation de la partie supérieure de cette courbe par un trapèze délimité par un trait horizontal au sommet et un trait de base prolongeant la droite d'ordonnée $\tau_{r}$. Cette schématisation est plus conforme à l'allure expérimentale réelle du diagramme et met en évidence en segment rectiligne initial entre 0 et $\tau_{r}$ auquel se rapporte pour les valeurs de $\mathrm{K}_{\mathrm{s}}$. Enfin l'aire du trapèze représente le travail développé par le système pour franchir la résistance de pic. Il y a lieu de se représenter, au cours des considérations qui suivront, combien ce travail est petit en comparaison à l'ensemble du diagramme intégral du système en régime de grandes déformations.
Plusieurs chercheurs ont procédé à des études approfondies sur la façon d'identifier les rugosités et l'influence qu'elles exercent sur le comportement mécanique. Sans amoindrir aucunement la valeur de ces recherches et des résultats expérimentaux obtenus, on doit néanmoins souligner l'influence déterminante de l'effet d'échelle dans ce domaine. Cette influence a été d'ailleurs déjà montrée expérimentalement par Barton. D'une façon plus générale, il suffira de remarquer que suivant les principes de la similitude mécanique, deux échantillons à différentes échelles géométriques constitués de la même matière et sujets aux mêmes contraintes, n'offrent la même résistance au cisaillement que si les rugosités sont elles-mêmes reproduites à l'échelle. II va de soi qu'en théorie un essai de cisaillement ne représente les conditions d'une surface entière de discontinuité intéressant un massif rocheux que si l'échantillon représente également, dans un certaine mesure, les effetș de rugosité réduits à l'échelle géométrique et mécanique, ce qu'il est presque impossible à réaliser.

On a remarqué en effet, que la nature de la roche et la nature des rugosités sont particulièrement déterminantes pour la valeur de $\tau_{p}$ ou, mieux, pour la différence $\tau_{p}-\tau_{r}$, ainsi que pour la valeur de $K_{s}$. 

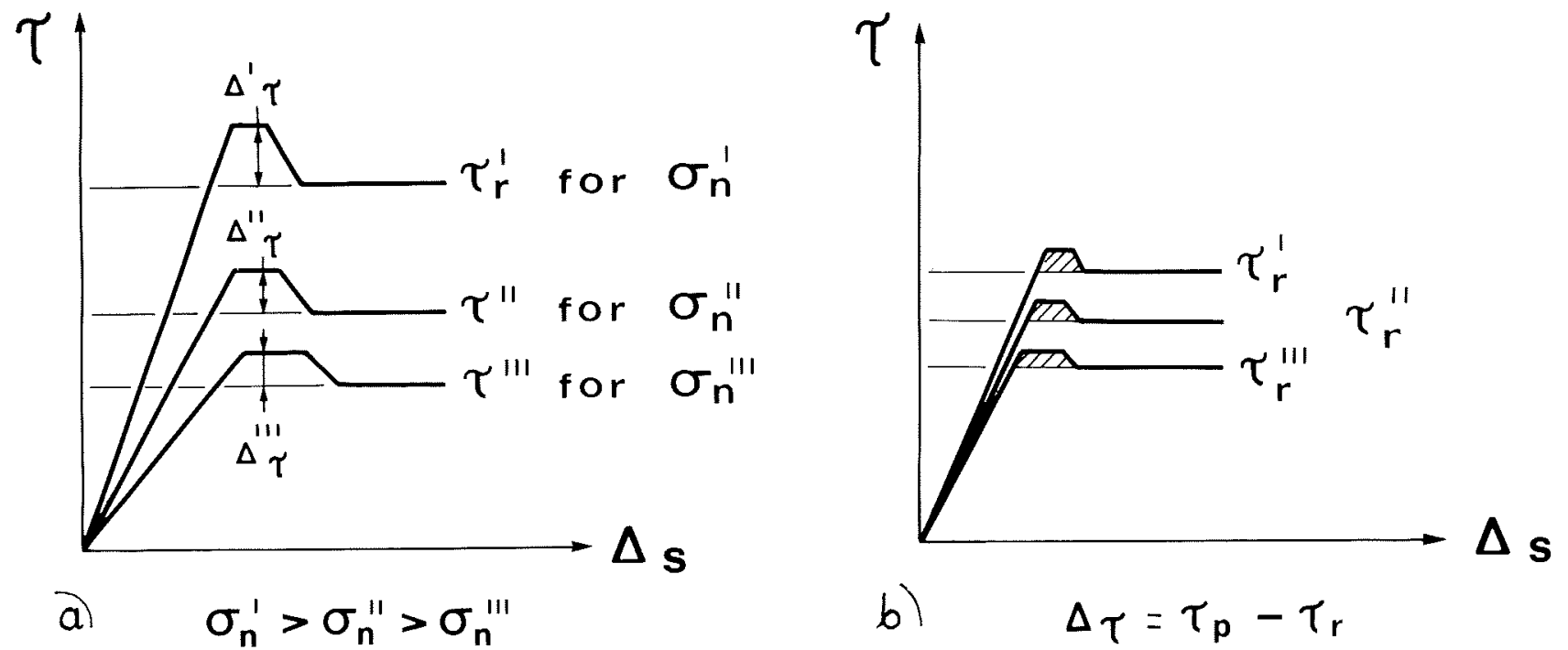

Fig. 3 Diagrammes représentatifs des essais de cisaillement pour des surfaces rugueuses (a) et des surfaces lisses (b)

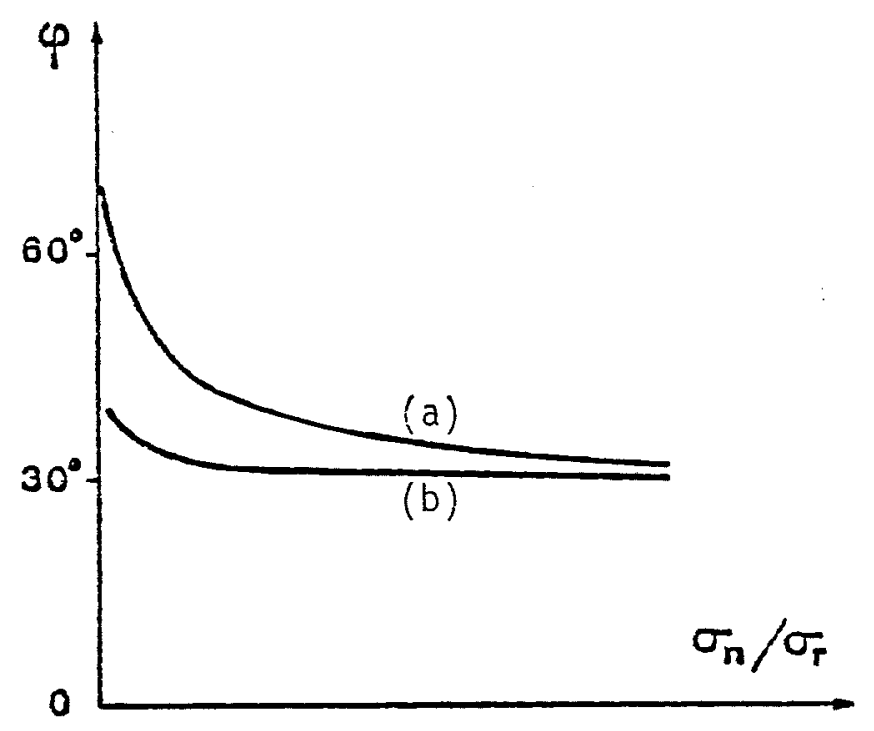

Fig. 4 Diagramme de variation de l'angle de frottement $\phi_{r}=f\left(\sigma_{n} / \sigma_{r}\right)$

(a) : surfaces rugueuses ; (b) : surfaces lisses
Les rugosités engendrent en effet des forces résistantes d'imbrication ou de cohésion apparente. Si les valeurs de $\sigma_{n}$ sont petites, par l'effet de la dilatation verticale et du détachement des surfaces, les rugosités sont surpassées; si par contre les valeurs de $\sigma_{n}$ augmentent, la dilatation entre les surfaces se réduit de plus en plus aux dépens d'un effet tranchant progressif des rugosités.

Or les rugosités - qui jouent un rôle important sur l'échantillon - deviennent des microrugosités si on les compare à l'étendue des plans de discontinuité dans un massif, et leur influence devient négligeable.

En fait, dans la réalité, les surfaces de discontinuité sont rarement d'une planéité parfaite sur de vastes étendues; bien souvent, elles sont masquées par des ondulations et des irrégularités d'ouverture et de rayon de courbure bien supérieures à celles qu'on peut reproduire sur échantillon. Leur influence est certes importante, mais leur accrochage est difficile à évaluer, même s'il est favorable à la stabilité du système.

Ce n'est que dans le cas de surfaces réduites lorsqu'on a affaire, par exemple, à des masses instables, à des coins de pénétration isolés, etc. - que les résultats obtenus sur échantillons peuvent s'avérer indicatifs (sans préjudice des considérations sur l'effet d'échelle) et que l'on peut utiliser, surtout pour les faibles valeurs de $\sigma_{n}$, des valeurs de $\phi_{r}$ nettement moins défavorables.

Par contre, dans le cas de surfaces très étendues, d'autres considérations déconseillent de tenir compte des valeurs de $\tau_{p}$ plutôt que de celles de $\tau_{r}$.

Considérons, à titre d'hypothèse, un plan de discontinuité très étendu, soumis à un cisaillement pur (fig. 5).

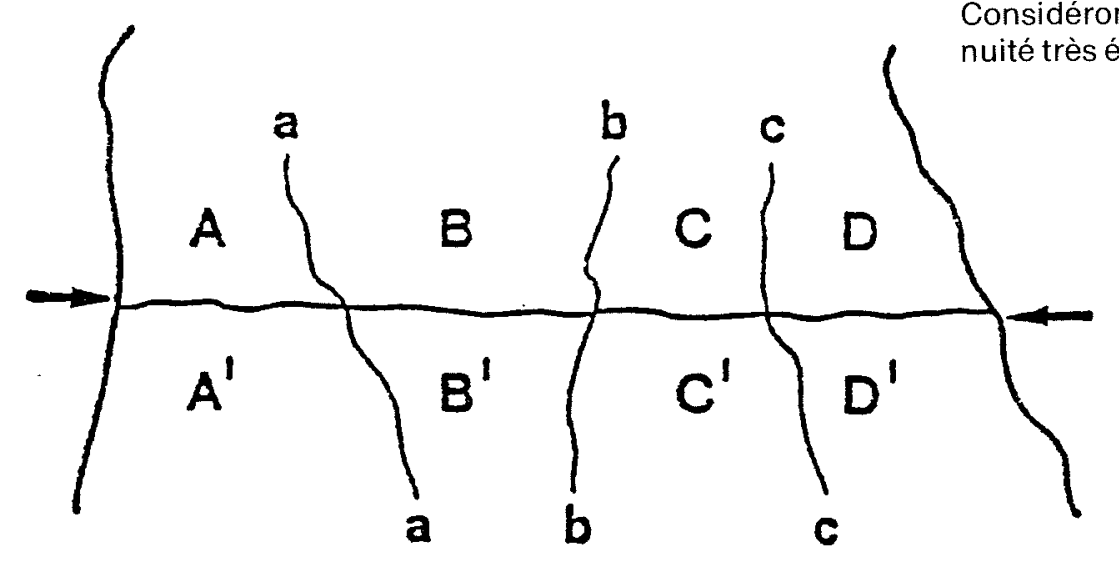




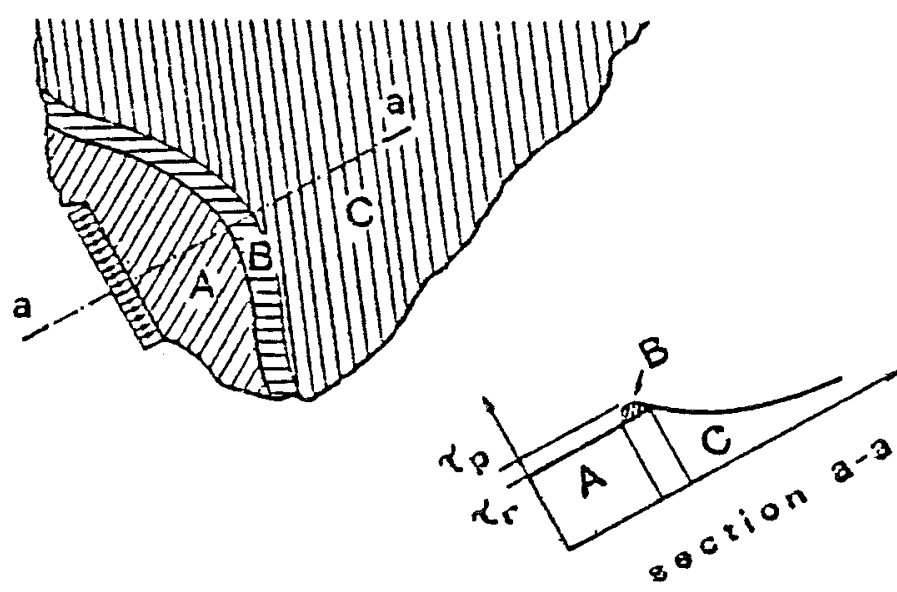

Fig. 6 Schéma de propagation de la rupture par cisaillement sous une surface de charge d'extension limitée A domaine en plasticité

B domaine du pic ou de limite élastique

C domaine en élasticité

Il existe souvent, dans un massif, d'autres ruptures ou discontinuités en intersection avec le plan en cours de sollicitation.

Le système ne peut guère rester parfaitement monolithique, de sorte que le glissement ne sera ni subit, ni général.

Si la fermeture initiale des joints a-b-c n'est pas totale, la répartition du cisaillement $n$ 'est pas homogène, $A$ et $\mathrm{D}$ n'étant pas en conditions de transmettre intégralement et de façon homogène les contraintes de cisaillement, de sorte que A glisse relativement par rapport à $A^{\prime}$ et $D$ par rapport à $D^{\prime}$, et qu'ils entrent en régime plastique $\left(\tau_{r}\right)$ avant que les surfaces $B-B^{\prime}$ et $C-C^{\prime}$ ne franchissent la résistance de pic $\left(\tau_{p}\right)$.

Nous pouvons donc supposer que la rupture par glissement n'est pas simultanée et qu'elle se transfère graduellement des blocs externes vers les blocs internes.

Dans la pratique, la situation est encore plus complexe. Considérons le cas plus général d'une surface de charge d'étendue limitée (un cas classique est représenté par la surface d'implantation d'un barrage) [fig. 6].

Les efforts de cisaillement diminuant au fur et à mesure de leur diffusion dans la masse, on peut supposer que la rupture par cisaillement débute à partir des zones superficielles soumises aux contraintes les plus élevées, franchit la résistance de pic $\left(\tau_{p}\right)$ et se propage à l'intérieur de la roche.

On aura alors une zone de plasticité limitée par une bande frontière en correspondance de laquelle le système rocheux développe la totalité de la résistance de pic $\left(\tau_{p}\right)$, pour décroître ensuite graduellement dans les zones les plus internes qui se trouvent encore en régime élastique.

Pour des charges plus élevées, la zone en régime plastique s'étend, les frontières résistantes du pic reculent progressivement jusqu'à la ligne d'affleurement extérieure, sur la surface opposée.

On peut penser que, dans la phase finale, les conditions de résistance limite se développent au sein du système (tout au moins dans l'hypothèse d'une résistance uniforme), c'est-à-dire quand la valeur $\tau_{\mathrm{p}}$ a été dépassée tout le long de la surface et que seule la résistance résiduelle $\tau_{r}$ agit.

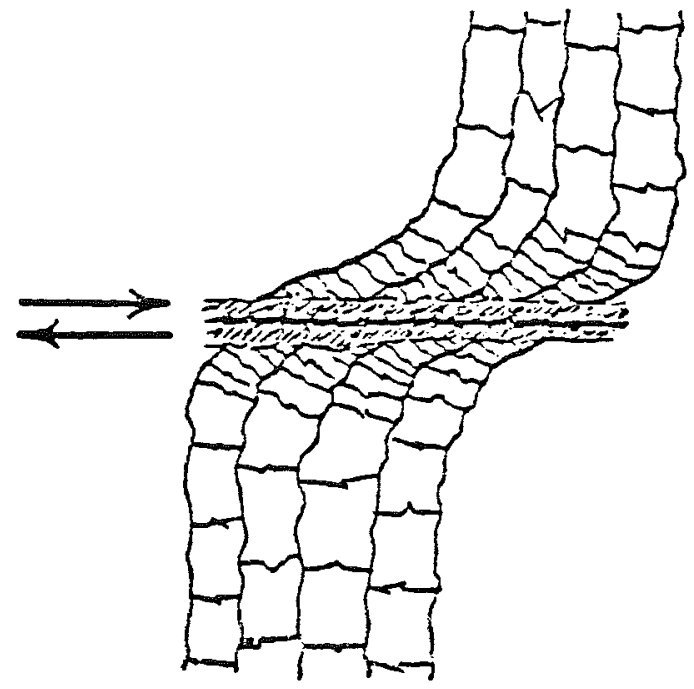

Fig. 7 Schéma des perturbations déterminées par une faille très active

Les considérations exposées soulignent qu'on ne saurait se baser sur la résistance $\tau_{p}$, et qu'il est prudent, pour une vérification sûre, de ne se rapporter qu'à la valeur de $\tau_{r}$ étendue à toute la surface en régime de plasticité.

Ces considérations sont suggérées et confirmées par les essais sur des modèles physiques.

\section{Considérations pratiques}

II faut maintenant se demander quels doivent être les critères de choix des valeurs de l'angle de frottement $\phi_{r}$.

Nous avons déjà constaté que cet angle diminue sensiblement lorsque $\sigma_{n}$ augmente et qu'on voit en même temps augmenter les ruptures par tranchement des rugosités superficielles.

Si la surface de discontinuité est une faille tectonique qui a subi des glissements très importants (fig. 7) en présence de tenseurs hydrauliques très élevés, on constate d'ordinaire la présence de plans de cisaillement spéculaires, accompagnés d'alternances chimico-physiques et structurales de la roche, avec inclusion, çà et là, de veines lubrificantes de mica, d'argile, de tourbe, de bitume et formation fréquente de bandes latérales de perturbations permettant de déceler le désordre local par des inflexions à l'inverse des couches et par la présence de matériaux très broyés.

Devant des phénomènes plus ou moins importants de cette nature, il faut procéder avec une extrême prudence, non seulement à cause de la valeur réduite de l'angle de frottement des discontinuités de cette origine, mais aussi parce qu'il y a lieu de soupçonner prudemment que ces failles sont encore potentiellement actives et qu'en pareil cas elles se dérobent à toute évaluation et à tout contrôle de sécurité. Elles peuvent constituer des «soupapes» de décharge de forces endogènes énormes qui s'accumulent souvent de façon continue dans le système rocheux.

Dans les cas les plus généraux, il sera bon d'évaluer l'ordre de grandeur de l'intensité des $\sigma_{n}$ agissant sur la surface, en y comprenant, si possible, les contraintes internes de la roche; et si la valeur de $\sigma_{n}$ ne dépasse 
pas 20 à $30 \%$ de la résistance propre de la roche matrice, les essais de cisaillement indiquent que pour le contact d'une roche saine en l'absence de toute inclusion, l'angle $\phi_{r}$ limite n'est jamais inférieur à $30^{\circ} \pm 2^{\circ}$.

Comme nous l'avons dit, c'est uniquement lorsque la valeur de $\sigma_{n}$ diminue que l'angle $\phi_{r}$ augmente graduellement, en fonction des rugosités résiduelles.

Néanmoins, dans le cas des surfaces lisses, $\phi_{r}$ ne dépasse jamais $34-35^{\circ}$; par contre, pour des surfaces très rugueuses, l'angle de pic $\phi_{\mathrm{p}}$ peut même dépasser $60-70^{\circ}$ (c'est le cas des équilibres par imbrication de blocs isolés en surface, sur des forts pendages).

En présence du contact de roche saine pour des surfaces de discontinuité assez étendues et des valeurs de $\sigma_{n}$ non négligeables (discontinuités à l'intérieur d'un massif), l'angle de frottement $\phi_{r}$ à adopter selon les critères prudents peut être évalué à $30^{\circ}$, au moins à titre d'approximation préliminaire.

Des angles de frottement plus élevés peuvent être adoptés à condition de connaître l'ordre de grandeur des $\sigma_{n}$ et de procéder à des essais de cisaillement pour déterminer les $\tau_{r}$ ainsi que l'influence des rugosités, compte tenu que, dans chaque cas, l'influence de ces rugosités est toujours sujette à l'effet d'échelle.

II reste à parler des méthodes indirectes mises au point pour évaluer les rugosités des surfaces de contact et l'influence de ces rugosités sur les essais de cisaillement.

Certains chercheurs (Rengers, Barton, etc.) ont entrepris des études approfondies sur cette question et ont obtenu des résultats technico-scientifiques certainement très valables. Néanmoins, dans le domaine des applications et sur la base des observations exposées, ces résultats ne semblent pas encore très prometteurs en ce qui concerne l'acquisition des paramètres nécessaires aux contrôles d'équilibre.

\section{Discontinuités avec intrusions}

Nous avons dit qu'il existait dans la nature des discontinuités d'un autre type, caractérisées par la présence d'intrusions de matériaux fort peu cohérents (argile, tourbe, bitume, mica, etc.) permettant, du fait de leur épaisseur, le glissement relatif sans points de contact rigide entre les surfaces de roche saine.

Dans le cas des tourbes et des argiles, le comportement dépend principalement - ce qui est intuitif - de la teneur en eau.

En cas d'argile très sèche, le comportement est caractéristiquement celui d'une roche à cohésion réduite; l'allure de la courbe de cisaillement et la valeur de $\tau_{r}$ ne diffèrent pas sensiblement de celle d'une roche cohérente. Dès que l'humidité intervient, $\mathrm{K}_{\mathrm{s}}$ se réduit, la zone de pic disparaît, $\tau_{r}$ diminue rapidement.

Le glissement est provoqué, en pareil cas, par un processus principalement visqueux, avec formation fréquente de pressions interstitielles.

En présence d'argile très humide, on rencontre en effet très souvent, même pour des contraintes normales $\sigma_{n}$ limitées à quelques dixièmes de $\mathrm{MPa}$, des angles de frottement de 6 à $7^{\circ}$. Étant donné le danger qu'implique ce phénomène, il nous reste à examiner attentivement si ces conditions doivent être envisagées comme des situations générales ou simplement comme des accidents locaux le long de la surface de discontinuité. a) 1 a 2 a 3 a

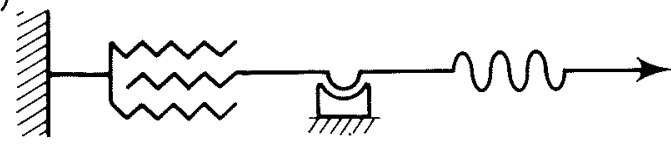

b)

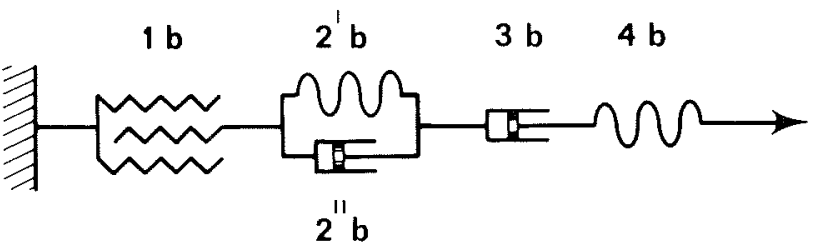

MECANISME DE DEFORMATION

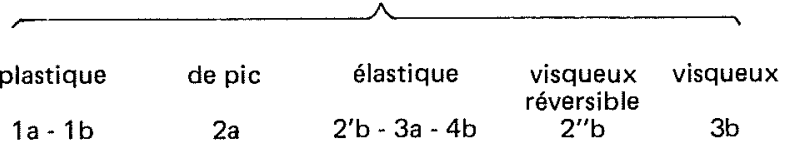

Fig. 8 Schéma rhéologique

(a) : modèle élasto-plastique; (b) : modèle élasto-plastique visqueux

Des valeurs déduites d'essais sur des échantillons non altérés peuvent même donner, dans des conditions plus favorables d'humidité, des valeurs de $\phi_{r}$ considérablement plus élevées; il faut toutefois se méfier des valeurs obtenues dans ces circonstances, le régime hydraulique dans la discontinuité pouvant varier en fonction d'un grand nombre de facteurs. Rappelonsnous en particulier, que les $\sigma_{n}$ peuvent donner lieu à des pressions interstitielles et à une décroissance rapide de l'angle $\phi_{r}$

\section{Schémas rhéologiques}

A titre de dernière indication, nous croyons pouvoir affirmer que la rupture par cisaillement entre des surfaces de roches saines peut être définie comme un processus éminemment élasto-plastique, avec des retours au déchargement relativement élastiques et instantanés.

Le modèle rhéologique qui peut le décrire est représenté par le schéma (a) de la figure 8 , tandis qu'en présence d'intrusions telles que de l'argile humide, le processus est de nature élasto-plastovisqueuse fortement influencé par la variable temps, conformément au schéma (b) de la figure 8.

Il est bon de remarquer également qu'il existe entre les valeurs des angles de cisaillement des systèmes (a) et (b) une différence qu'on peut estimer, à titre indicatif, entre 15 et $28^{\circ}$, et qu'il est rare de rencontrer dans la nature. 


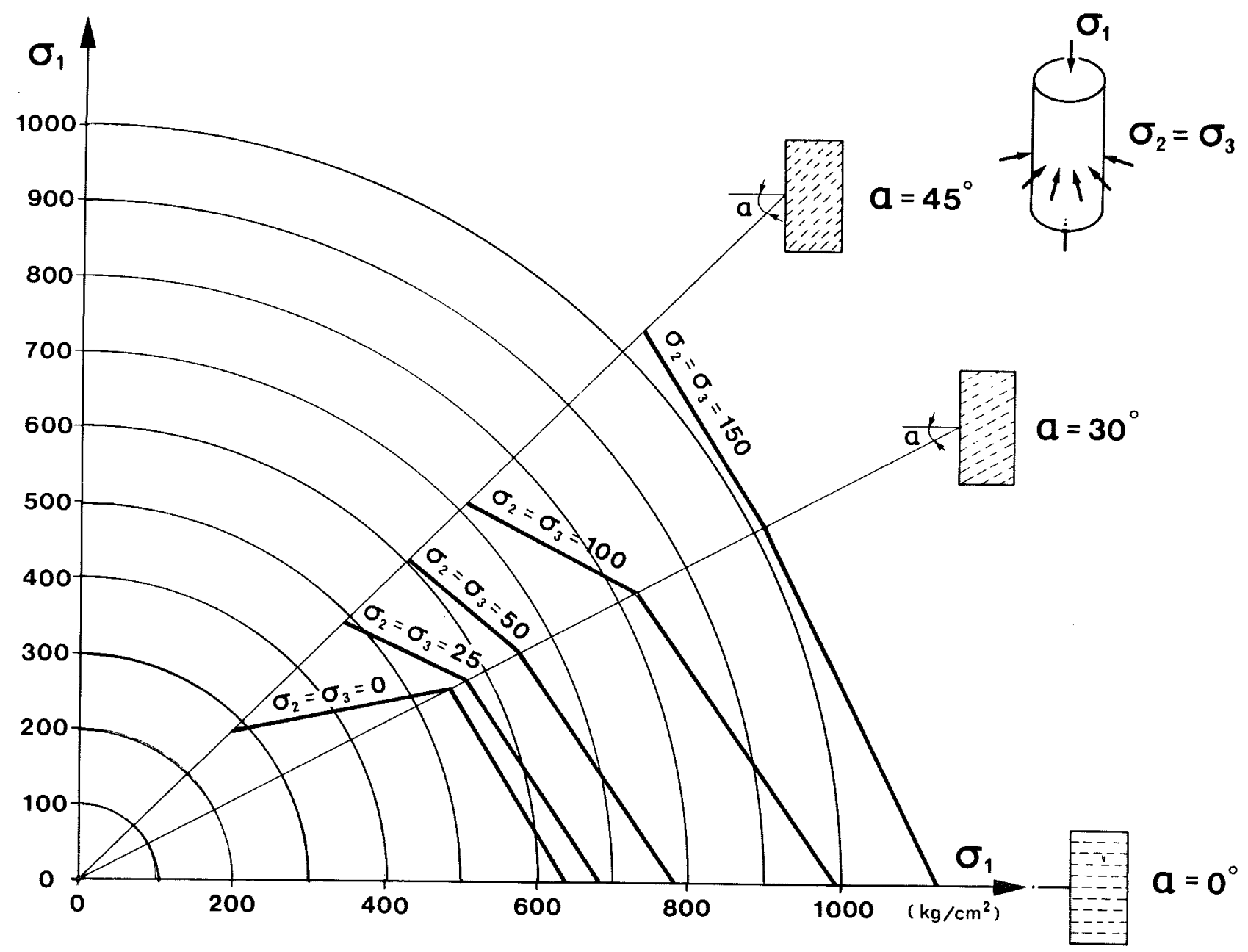

Fig. 9 Diagrammes des résistances triaxiales déterminées sur échantillons cylindriques pour différentes orientations des discontinuités dans la roche

\section{Discontinuités hétérogènes}

Il est des cas où les surfaces de roche saine ne se trouvent en contact réciproque que par endroits, l'aire de discontinuité contenant des intrusions lenticulaires.

II y a lieu de remarquer, dans de pareils cas, que la valeur de $\phi_{\mathrm{r}}$ ne doit pas être évaluée comme une valeur moyenne, en tenant compte du pourcentage des surfaces avec ou sans intrusions, car les zones de contact en roche saine s'opposent fortement au glissement selon les caractéristiques qui leur sont propres, attirant sur elles le flux des contraintes.

L'angle $\phi_{r}$ doit être alors évalué comme étant relativement proche de celui de la roche saine du contact. II faudra considérer, tout au plus, que les valeurs de $\sigma_{n}$ et de $\phi_{r}$ agissant sur les zones à contact solide sont en moyenne plus élevées et que leur flux est troublé par des perturbations et distorsions locales. Dans de pareils cas les essais in situ et en laboratoire sont extrêmement délicats et leurs résultats sont souvent très peu croyables.

\section{Quelques considérations finales}

Tout ce que nous venons d'exposer souligne la difficulté de fournir des indications de valeur générale.
D'après ce qui vient d'être dit, on peut affirmer que les valeurs des essais effectués - in situ ou au laboratoire - par des méthodes simplifiées sont généralement très peu dignes de foi. Seuls des essais conduits avec soin, menés à bien par des moyens appropriés et selon des technologies avancées peuvent être acceptés. Des essais grossiers et des critères d'interprétation approximatifs n'éclairent pas mieux que ne le ferait une évaluation à priori de la part d'un technicien suffisamment expérimenté. On s'adresse parfois à des essais de cisaillement sur des éprouvettes monolithiques pour la détermination de la cohésion et de l'angle de frottement de la roche elle-même en tant que paramètres de la loi de Coulomb. Il faut alors se rappeler que si les modalités de rupture s'éloignent sensiblement du plan théorique de glissement les résultats qu'on peut obtenir ne sont guère dignes de foi. A ce sujet, les résultats d'une série d'essais en cellule triaxiale sur éprouvettes cylindriques peuvent s'avérer beaucoup plus corrects et probants. La variation de la tangente à la courbe enveloppe aux cercles de rupture $\sigma_{1}^{\prime}-\sigma_{3}^{\prime}$ selon la représentation de Mohr peut fournir des indications, peut-être plus correctes, sur la variation de l'angle $\phi_{r}=f\left(\sigma_{n}\right)$. Enfin, en présence de plans de discontinuité, il est possible d'identifier la chute de résistance en fonction de l'orientation des plans par rapport au tenseur des contraintes (fig. 9). 
Dans le cas de discontinuités entre roches à contact cohérent, la courbe $\phi_{r}=f\left(\sigma_{n}\right)$ tend vers une valeur minimale qu'on peut évaluer à $30^{\circ} \pm 2^{\circ}$ tout au moins pour des valeurs de $\sigma_{n}$ suffisamment grandes, telles qu'on peut les rencontrer en pratique dans les ouvrages de génie civil.

Les rugosités jouent un rôle aléatoire très important lorsqu'on opère sur des surfaces d'étendue réduite; les forces d'imbrication revêtent en pareil cas une valeur déterminante pour l'angle $\phi_{r}$ lorsque les valeurs de $\sigma_{n}$ sont faibles. L'influence de ces rugosités doit être considérée par contre comme négligeable, par le jeu de l'effet d'échelle, pour des surfaces très étendues faisant partie d'un massif rocheux. Les résistances de cohésion dues à une cimentation locale et intermittente le long des surfaces de discontinuité exercent, lorsqu'elles sont loin de reproduire une liaison monolithique pour la continuité et la résistance, des effets comparables à la rugosité; en d'autre termes, elles sont influentes seulement pour des surfaces d'importance réduite et pour des valeurs faibles de $\sigma_{n}$.
Les exigences du calcul amènent aujourd'hui à déterminer certains paramètres, notamment la valeur de $\mathrm{K}_{\mathrm{s}}$. Sans vouloir aucunement nier l'intérêt d'une pareille expérimentation, il faut souligner, dans chaque cas, que les courbes $\tau-\Delta_{s}$ obtenues sont influencées, dans une large mesure, par les aléas des éprouvettes et s'avèrent, de ce fait, très dispersées. En tout cas, l'influence de $\sigma_{n}$ et de la vitesse d'accroissement des cisaillements est déterminante sur l'allure des diagrammes et sur les valeurs de $\mathrm{K}_{\mathrm{s}}$.

En présence d'intrusion de tourbe et d'argile dans les discontinuités, il y a lieu de considérer comme valables les propositions extrêmement prudentes déjà exposées. En pareil cas, toute évaluation doit prendre en compte ces intrusions, surtout si une modification de ce régime est possible par exemple sous l'effet d'infiltrations lors de l'exécution d'ouvrages hydrauliques. 
